\title{
Mechanism of Ultra-Fast Actin-Myosin Sliding Producing Cytoplasmic Streaming in Giant Algal Cell, Studied Using the Centrifuge Microscope
}

\author{
Shigeru Chaen ${ }^{1}$ and Haruo Sugi ${ }^{2 *}$ \\ ${ }^{1}$ Department of Biosciences, College of Humanities and Sciences, Nihon University, Tokyo, Japan \\ ${ }^{2}$ Department of Physiology, School of Medicine, Teikyo University, Tokyo, Japan
}

\begin{abstract}
In giant intermodal cells of green algae Chara collaria, cytoplasmic streaming is produced by ATP-dependent sliding between myosin heads extending from amorphous cytoplasmic organelles and actin filament arrays (actin cables) fixed on chloroplast rows. The velocity of cytoplasmic streaming is many times faster than the maximum myofilament sliding in skeletal muscle. In this article, we compared steady-state force-velocity (P-V) relations between cytoplasmic myosin and skeletal and cardiac muscle myosins using the centrifuge microscope, in which myosincoated latex beads were made to slide along the actin cables under various centrifugal forces. In contrast with the hyperbolic P-V relation of actin-myosin sliding in skeletal and cardiac myosins, the P-V relation of cytoplasmic myosin versus actin cable sliding was a straight line, indicating a very large duty ratio and a very small rate of chemomechanical energy conversion. Possible mechanisms of the ultra-fast actin-myosin sliding are discussed.
\end{abstract}

Highlights

- The velocity of cytoplasmic streaming, caused by ATP-dependent sliding between cytoplasmic myosin and actin cables in giant algal cells is many times faster than ATP-dependent actin-myosin sliding in skeletal and cardiac muscles.

- The mechanism of ultra-fast actin-myosin sliding was studied using the centrifuge microscope, in which beads coated with cytoplasmic myosin were made to slide along actin cables under various centrifugal forces serving as loads against cytoplasmic myosin versus actin cable sliding

- Unlike the hyperbolic force-velocity (P-V) relation of skeletal and cardiac muscle actin-myosin sliding, the P-V relation of cytoplasmic actin myosin sliding was a straight line irrespective of the force generated by cytoplasmic myosin.

- These results indicate a very large duty ratio and a very small efficiency of chemo-mechanical energy conversion in cytoplasmic actin-myosin sliding.

Keywords: Ultra-fast actin-myosin sliding; Cytoplasmic streaming; Giant algal cell; Force-velocity relation; Centrifuge microscope; Maximum shortening velocity

\section{Characteristic Features of ATP-Dependent Actin-Myosin Interaction Producing Cytoplasmic Streaming}

Cytoplasmic streaming is widely observed in plant cells. The mechanism of cytoplasmic streaming has been investigated on giant intermodal cells of green algae Chara collaria (diameter, $\sim 0.5 \mathrm{~mm}$; length, $>10 \mathrm{~cm}$ ) (Figure 1) with the following results: (1) The motive force for Chara cytoplasmic streaming is generated by ATP-dependent interaction between Chara cytoplasmic myosin molecules extending from amorphous cytoplasmic organelles and well-organized straight actin filament arrays fixed on chloroplast rows, called actin cables (Figure 2); (2) The direction of streaming is reversed across the indifferent zone where chloroplast is absent, reflecting the reversal of actin filament polarity across the indifferent zone; (3) Since the two indifferent zones run parallel to the intermodal cell long axis, cytoplasmic streaming rotates longitudinally within the cell [1-7].

Although both cytoplasmic streaming and muscle contraction are produced by ATP-dependent actin-myosin sliding, and regulated by intracellular $\mathrm{Ca}$ ion concentration, skeletal muscle is relaxed at $\mathrm{pCa} \geq$ 7 and fully contracts at $\mathrm{pCa} \leq 5$, while cytoplasmic streaming occurs at $\mathrm{pCa} \geq 7$, and stops at $\mathrm{pCa} \leq 6[8-10]$. If small beads coated with skeletal muscle myosin is made to slide along actin cables in the presence of ATP, the bead movement is insensitive to pCa [11], indicating the absence of regulatory proteins in actin cables. On the other hand, the stoppage of cytoplasmic streaming is caused by tight cytoplasmic myosin-actin cable linkages, which is stronger than their rigor linkages in the absence of ATP, suggesting that Chara cytoplasmic myosin can bind with actin cables in two different modes.

The most interesting feature of cytoplasmic streaming in giant intermodal cells is that its velocity $(\geq 50 \mu \mathrm{m} / \mathrm{s})$ [1] is many times larger than the maximum velocity of skeletal muscle shortening, i.e. siding between actin and myosin filaments $(\leq 10 \mu \mathrm{m} / \mathrm{s})$. In the presence of ATP, beads coated with skeletal muscle myosin move on actin cables at velocities similar to that of actin-myosin sliding in muscle [12,13]. In contrast, skeletal muscle actin filaments move on a glass surface coated with Chara cytoplasmic myosin at velocities similar to that of native cytoplasmic streaming [14]. These results indicate that it is Chara cytoplasmic myosin that causes the ultra-fast velocity of cytoplasmic

*Corresponding author: Haruo Sugi, Department of Physiology, School of Medicine, Teikyo University, Tokyo, Japan, Tel: +81 48478 4079; E-mail: sugi@kyf.biglobe.ne.jp

Received January 10, 2017; Accepted February 13, 2018; Published February 23, 2018

Citation: Chaen S, Sugi H (2018) Mechanism of Ultra-Fast Actin-Myosin Sliding Producing Cytoplasmic Streaming in Giant Algal Cell, Studied Using the Centrifuge Microscope. J Material Sci Eng 7: 426. doi: 10.4172/2169-0022.1000426

Copyright: $\odot 2018$ Chaen S, et al. This is an open-access article distributed under the terms of the Creative Commons Attribution License, which permits unrestricted use, distribution, and reproduction in any medium, provided the original author and source are credited. 


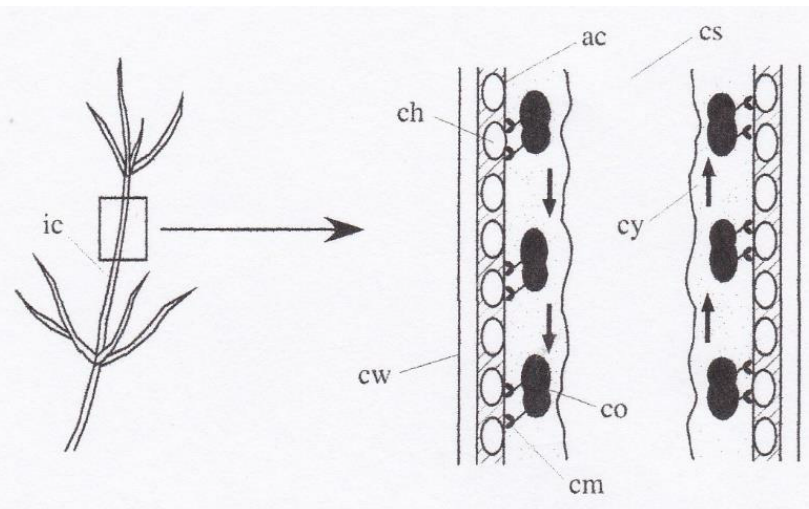

Figure 1: Diagram showing cytoplasmic streaming in giant intermodal cell of green algae Chara corallina. ac, actin cables; ch, chloroplast; $\mathrm{cm}$, cytoplasmic myosin; co, cytoplasmic organelle; cs, cell sap; cw, cell wall; cy, streaming cytoplasm, Arrows indicate direction of streaming; ic, intermodal cell.

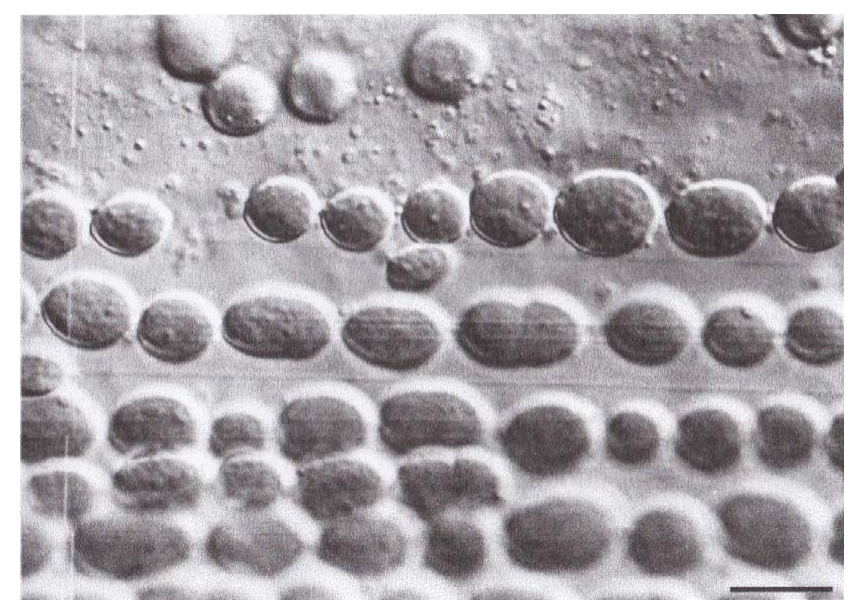

Figure 2: Actin cables on the inner surface of chloroplast rows in an intermoda cell of Chara corallina. Calibration bar, $10 \mu \mathrm{m}[7]$. streaming. As with skeletal muscle myosin, Chara cytoplasmic myosin molecule is a dimer consisting of two heavy chains with one head and one tail, so that they have two heads and one helical tail, but the tail length is much shorter than that in skeletal muscle myosin molecules [15].

\section{Centrifuge Microscope to Study Steady-state Force- velocity Relation of ATP-dependent Sliding between Myosin and Actin Cables}

Figure 3 illustrates the setup of the centrifuge microscope constructed in our laboratory [13]. It consisted of a light microscope, a rotor, and a stroboscopic light source. The specimen used was a short segment of an intermodal cell of green algae, into which myosin-coated polystyrene beads (diameter, $2.8 \mu \mathrm{m}$; specific gravity, 1.3) were introduced together with experimental solution containing MgATP. The specimen was placed in a cuvette, fixed on the rotor of the centrifuge microscope, in such a way that actin cables were parallel to the direction of centrifugal forces applied. Centrifugal forces directed opposite to the bead movement served as positive external loads on myosin molecules producing actin-myosin sliding. The amount of centrifugal force (=load) F on the bead is given by:

$$
\mathrm{F}=\Delta \rho \operatorname{Vr} \omega^{2} \text {, }
$$

Where $\Delta \rho$ is the difference in density between the bead and the surrounding medium $\left(0.3 \mathrm{gcm}^{-3}, \mathrm{~V}\right.$ is the bead volume $\left(12 \mu \mathrm{m}^{3}\right), \mathrm{r}$ is the effective radius of centrifugation $(4.5-7 \mathrm{~cm})$ and $\omega$ is the angular velocity of the rotor. Further details of the centrifuge microscope have been described elsewhere [13]. Experiments were made at $20-23^{\circ} \mathrm{C}$.

\section{Steady-state Force-velocity Relation of ATP-dependent Sliding between Skeletal Muscle Myosin and Actin Cables}

First, we will describe steady-state force-velocity relation of ATPdependent sliding between skeletal muscle myosin, prepared from rabbit psoas muscle, and actin cables. Under a constant centrifugal force serving as a positive external load, myosin-coated beads are

B

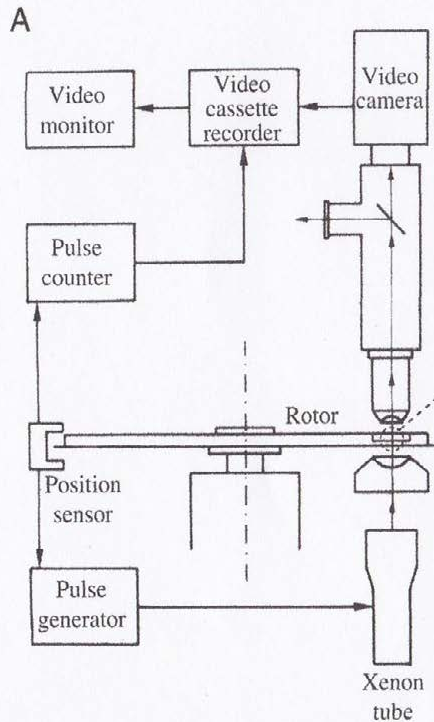

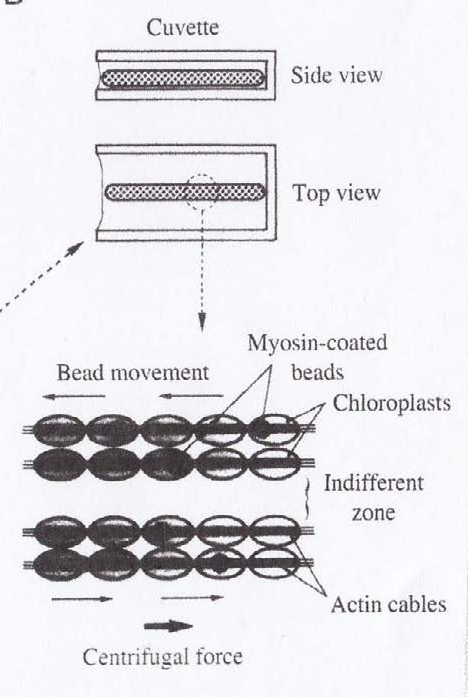

Figure 3: Centrifuge microscope for studying ATP-dependent sliding between myosin-coated beads and actin cables. (A) Diagram showing centrifuge microscope and video recording system. (B) Application of centrifugal forces serving as positive loads on the sliding beads [13]. 
Citation: Chaen S, Sugi H (2018) Mechanism of Ultra-Fast Actin-Myosin Sliding Producing Cytoplasmic Streaming in Giant Algal Cell, Studied Using the Centrifuge Microscope. J Material Sci Eng 7: 426. doi: 10.4172/2169-0022.1000426

observed to move along chloroplast rows (Figure 4) [13,16]. The beads continue moving with constant velocities determined by the amount of external load over a long distance, indicating the steady state between the load (=force $\mathrm{P}$ ), generated by myosin molecules on the bead and the velocity of actin-myosin sliding (Figure 5A). The maximum unloaded velocity of bead movement was $1.6-3.6 \mu \mathrm{m} / \mathrm{s}$. The bead stopped moving when the load was increased to the maximum isometric force Po generated by myosin molecules on the bead. As shown in Figure 5B, the force-velocity $(\mathrm{P}-\mathrm{V})$ curve of the bead movement with high Po values $(10-39 \mathrm{pN})$ was hyperbolic in shape in the low force region, but deviated from hyperbola in the high force region. The shape of the P-V curve was nearly similar to that obtained from tetanized single skeletal muscle fibers $[17,18]$, and $\mathrm{Ca}^{2+}$-activated single skinned muscle fibers (Figure 6) $[19,20]$. These results not only indicate that skeletal muscle myosin molecules on the bead retain their basic kinetic properties of contracting muscle, but also close similarities between actin cables and skeletal muscle actin filaments.

Meanwhile, in the P-V curves of the bead movement with low Po values (3.5-5.9 $\mathrm{pN})$, the hyperbolic part at low force region became much less pronounced (Figure 7). Similar results were obtained on the $\mathrm{P}-\mathrm{V}$ curves of sliding movement of the beads coated with two different myosin isoforms, V1 and V3, obtained from rabbit cardiac muscle [21]. In this study, we made a tentative explanation for the increasing

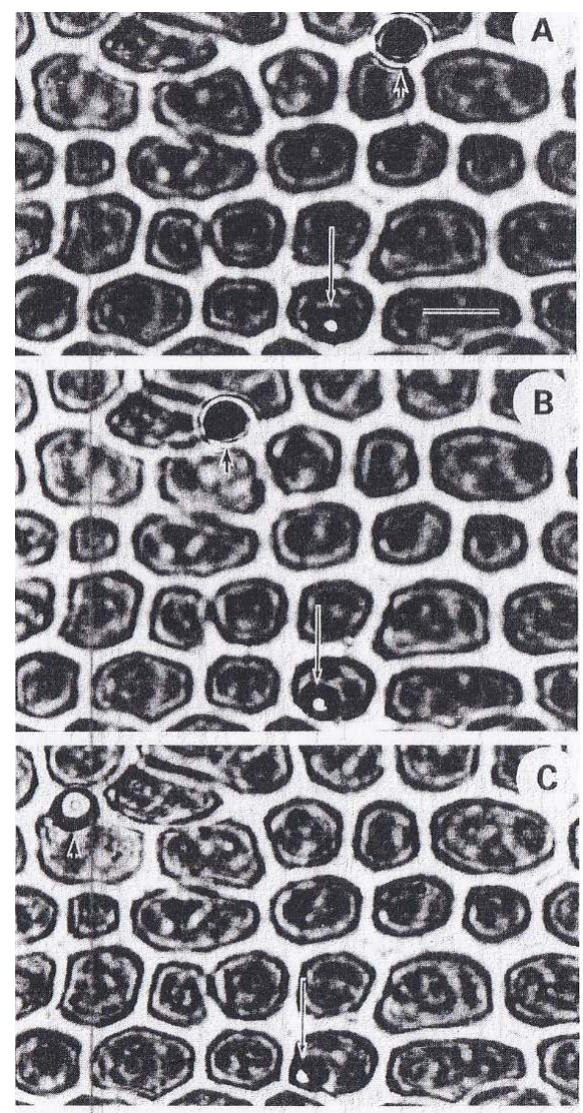

Figure 4: Selected video frames showing two myosin-coated polystyrene beads sliding from right to left along $h$ chloroplast rows, on which actin cables (not visible) run straight. One bead (diameter, $4.5 \mu \mathrm{m}$, upper edge of each frame, indicated by upward arrows) is coated with skeletal muscle myosin, while the other bead (diameter, $2.8 \mu \mathrm{m}$, lower edge of each frame, indicated by downward arrows) are coated with skeletal muscle myosin previously treated anti-subfragment 2 antibody [16].
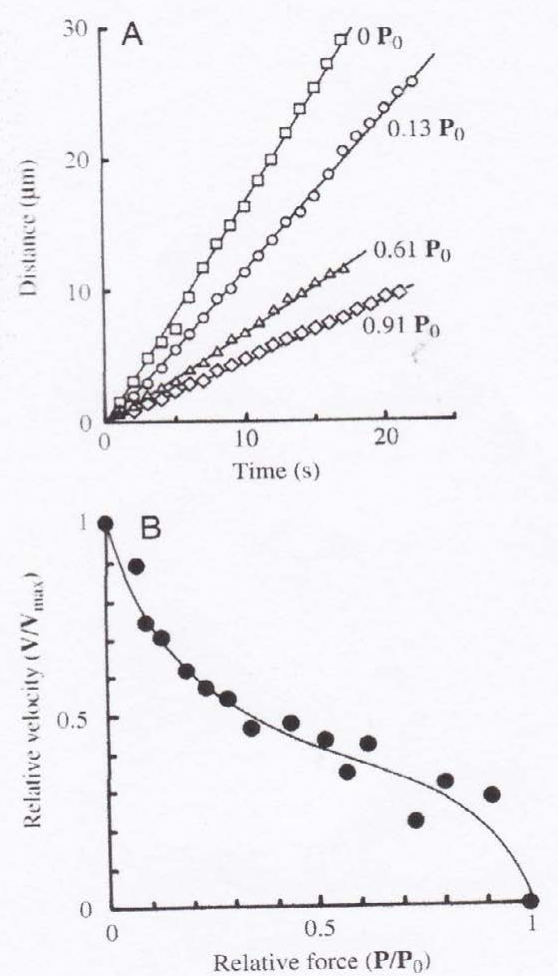

Figure 5: Force-velocity (P-V) relation of ATP-dependent sliding between beads coated with skeletal muscle myosin and actin cables with high Po values. (A) Constant velocity sliding of a skeletal muscle myosin-coated bead on actin cables under 4 different centrifugal forces, serving as positive loads expressed relative to the maximum isometric force $\mathrm{Po}(15 \mathrm{pN})$. (B) P-V curve of skeletal muscle myosin versus actin cables, obtained from the records in (A). Both velocities and forces are expressed relative to the maximum values [13].

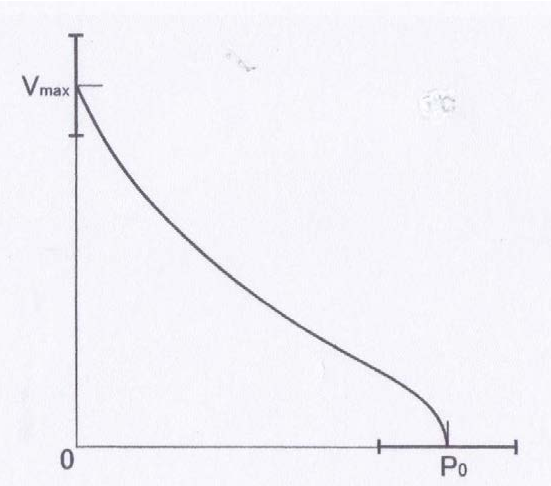

Figure 6: Averaged P-V curve of single skinned skeletal muscle fibers obtained from 6 different fibers. Note that the curve deviates from the hyperbola in the high force region [20].

deviation of P-V curves from the hyperbola with decreasing Po in the following way. The ATP-dependent-actin-myosin sliding is caused by cyclic attachment and detachment between myosin heads and actin filaments. According to the Huxley contraction model [22], the probability that a myosin head is attached to actin is $0<p<1$, and the probability that all myosin heads interacting with actin are in detached state, i.e. off probability Poff is given by: 


$$
\text { Poff }=(1-p)^{\mathrm{N}}
$$

where $\mathrm{N}$ is the number of myosin heads interacting with actin. As shown in Figure 8, Poff becomes substantial for small N. During the period of Poff, Beads are displaced in the direction opposite to that of actin-myosin sliding, to result in retardation of the observed sliding velocity under the centrifuge microscope. Assuming the appropriate dependence of $p$ on the amount of load for cardiac myosin isoforms $\mathrm{V} 1$ and V3, the shortening velocities in the small load (=force) region of P-V curves with small Po values are corrected to be larger than the observed values. This implies that, if the number of myosin heads interacting with actin is small, the shortening velocity in the small load region is slowed down due to increase in the moment, at which all myosin heads detach from actin.

In Figure 9, broken lines indicate P-V curves for $\mathrm{V} 1$ myosin(A) and for V2 myosin actually obtained by the centrifuge microscope, while solid curves indicate $\mathrm{P}-\mathrm{V}$ curves corrected on the assumption that Poff $=0$ for all load (=force) region. It can be seen that the marked deviation from the hyperbola of the P-V curve with low Po values (broken lines, cf. Figure 7) can be made much less pronounced (solid lines) to resemble the P-V curve with high Po values (cf. Figure 5B).

\section{Steady-state Force-velocity Relation of ATP-dependent Sliding between Cytoplasmic Myosin and Actin Cables}

During the course of experiments with skeletal muscle myosin, we found that, when uncoated beads were introduced into the giant

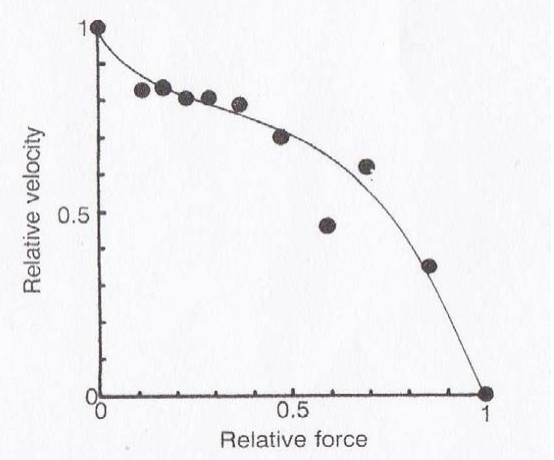

Figure 7: P-V curve of skeletal muscle myosin versus actin cables with low Po values. Both velocities and forces are expressed relative to the maximum values. Note that the hyperbolic part of the curve at low force region is much less pronounced compared to the P-V curve shown in Figure 5B [13].

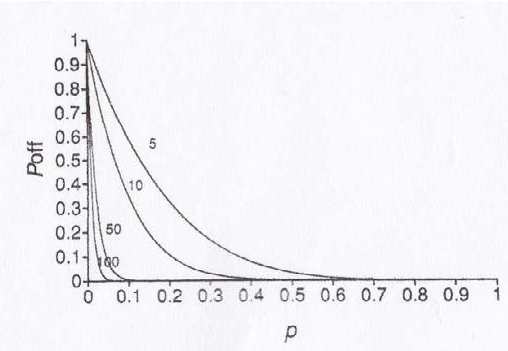

Figure 8: Dependence of Poff, i.e. the probability that all myosin heads interacting with actin are detached at any chosen moment, on the fraction of time, in which a myosin head is attached firmly to actin within one cycle of attachment-detachment cycle $p(0<p<1)$. Numbers alongside each curve indicate number of myosin heads interacting with actin [21].
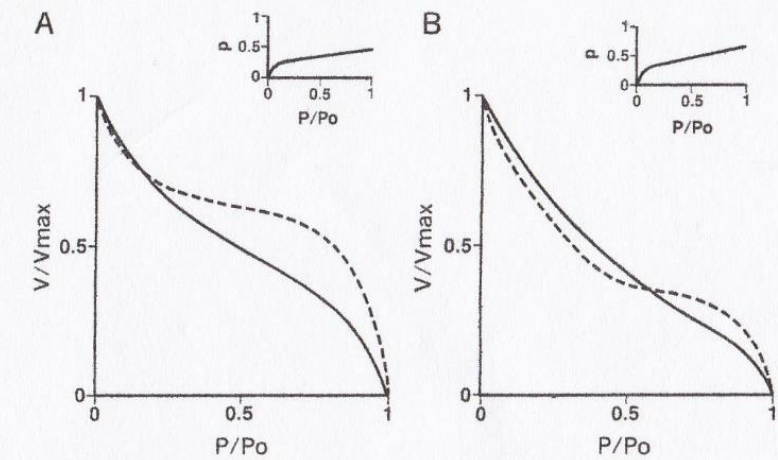

Figure 9: Corrected P-V curves (solid lines) for two different kinds of vertebrate cardiac muscle myosin isoforms ( $\mathrm{A}$ for $\mathrm{V} 1$ myosin and $\mathrm{B}$ for $\mathrm{V} 2$ myosin). Broken lines indicate P-V curves obtained experimentally from 6 different fibers. Both velocities and forces are expressed relative to the maximum values. Dependence of $p$ on external load for V1 and V2 myosins are also shown (insets). Note that the hyperbolic part of the curve becomes more pronounced after correction of the P-V curves based on Poff [21].

internodal cell preparation, the bead always started moving along actin cables with velocities similar to those of native cytoplasmic streaming. This phenomenon was explained as being due to Chara cytoplasmic myosin molecules, which had been detached from cytoplasmic organelles and remained in the intermodal cell preparation; such Chara myosin molecules attach to the uncoated bead surface, in such a way to produce cytoplasmic streaming in the same manner when they attach to cytoplasmic organelles. Therefore, we took the opportunity to study P-V curves of sliding movement of Chara myosin-coated beads along actin cables [23].

As has been the case in skeletal and cardiac muscle myosins, beads spontaneously covered with Chara cytoplasmic myosin, which will hereafter be simply called cytoplasmic myosin, moved with constant velocities under various centrifugal forces serving as external loads, indicating a definite steady-state between the force (=load) generated by cytoplasmic myosin and the velocity of actin-myosin sliding (Figure 10). The velocity of bead movement decreased with increasing external load, and the bead eventually stopped moving when the load became equal to the maximum isometric force Po generated by cytoplasmic myosin molecules. The beads that stopped moving remained in the same position on actin cables for 5-10 s and then suddenly detached from actin cables to flow away out of microscopic field. The value of Po showed a wide range of variation from 1.0 to $13 \mathrm{pN}$ (mean \pm SD, $6.2 \pm$ $4.5 \mathrm{pN}, \mathrm{n}=12$ ).

The steady-state P-V curves of the ATP-dependent bead movement along actin cables were obtained either by gradually increasing external load or by randomly changing external load with similar results. A typical example of the $\mathrm{P}-\mathrm{V}$ relation obtained from a bead $\left(\mathrm{Po}_{\mathrm{o}}=13 \mathrm{pN}\right)$ is shown in Figure 11 (filled circles). The P-V curve was nearly straight in shape. The viscous drag force $\mathrm{F}$ on the bead can be calculated as:

\section{$\mathrm{F}=6 \pi \mathrm{r} \eta v$,}

Where $\eta$ is the viscosity of the medium $\left(0.001 \mathrm{Ns} / \mathrm{m}^{2}\right), \mathrm{r}$ is the bead radius $(1.4 \mu \mathrm{m}$ ) and $v$ is the bead velocity (in $\mu \mathrm{m} / \mathrm{s}$ ). For $\nu=60 \mu \mathrm{m} / \mathrm{s}$, for example, the drag force is $1.6 \mathrm{pN}$, amounting to more than $10 \%$ of measured value of Po. In Figure 11, the data points corrected for the viscous drag are also shown by open circles. This correction does not alter the shape of the P-V curve obtained. Similar nearly straight $\mathrm{P}-\mathrm{V}$ curves were obtained from 6 other beads with high Po values (8.6- 


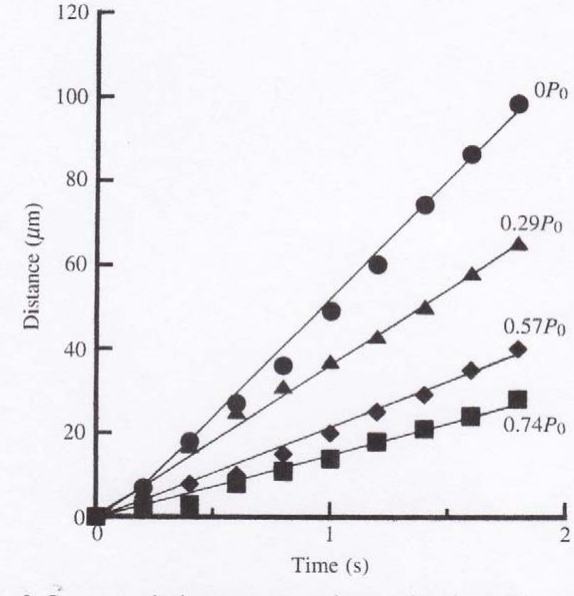

Figure 10: Constant velocity sliding of a bead, with cytoplasmic myosin attached, along actin cables under different positive loads, expressed relative to the maximum isometric force $\mathrm{Po}(13 \mathrm{pN})[23]$

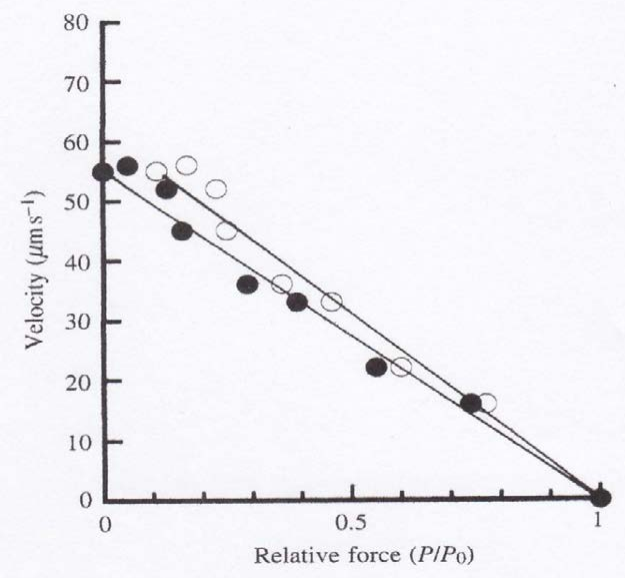

Figure 11: Steady-state P-V curve of a bead, with cytoplasmic myosin attached, on actin cables (filled circles). The loads were applied in a random order. The amount of force (=load) is expressed relative to Po $(13 \mathrm{pN})$. Data points corrected for the viscous drag force (open circles) are also shown [23].

$13 \mathrm{pN}$ ). Figure 12 shows averaged P-V relation constructed from 6 different beads with high Po values [23].

Figure 13 is averaged P-V curve constructed from 6 different beads with small Po values (1.0-2.4 pN), which may reflect extremely small numbers of cytoplasmic myosin molecules producing bead movement. Although the data points showed a large scatter, especially at high load region, the overall P-V curve was nearly straight [23].

\section{Kinetic Properties of Cytoplasmic Myosin Heads Producing the Ultra-fast Cytoplasmic Streaming}

The hyperbolic P-V curve of contracting whole skeletal muscle is fitted to part of rectangular hyperbola as:

$$
(\mathrm{P}+\mathrm{a}) \mathrm{V}=\mathrm{b}(\mathrm{Po}-\mathrm{P}),
$$

where $\mathrm{P}$ is the load on muscle, Po is the maximum isometric force generated by muscle, and $\mathrm{a}$ and $\mathrm{b}$ are constants. The P-V curve is generally characterized by values of the maximum unloaded shortening

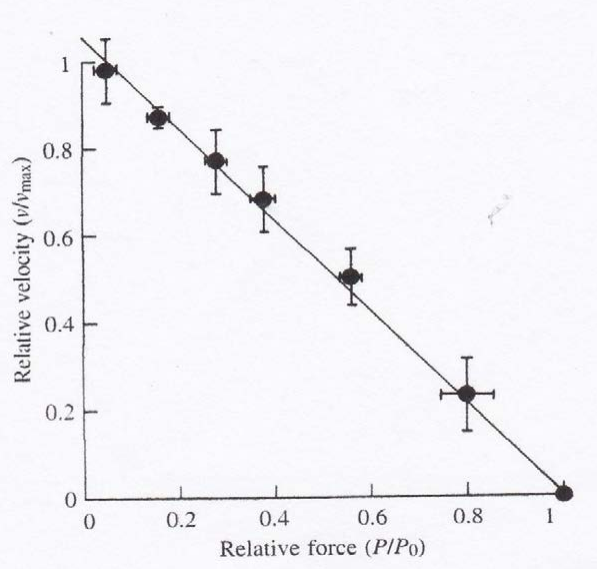

Figure 12: Steady-state $P-V$ relation of cytoplasmic myosin versus actin cable, constructed from 6 different beads, with large Po values $(8.6-13 \mathrm{pN})$. Bars indicate SD $(n=6)$. Note that the $P-V$ relation is straight [23].

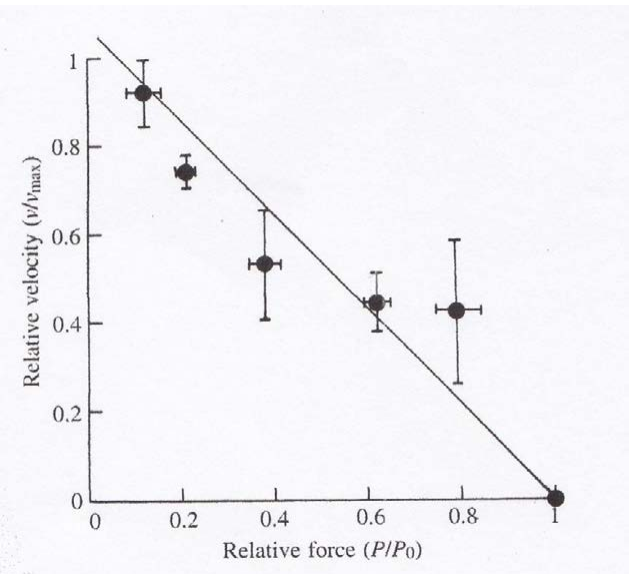

Figure 13: Steady-state $P-V$ relation of cytoplasmic myosin versus actin cable, Constructed from 6 different beads with small Po values $(1.0-2.4 \mathrm{pN})$. Bars indicate SD $(n=6)$ [23].

velocity Vmax and a/Po [24]; the former determines its intercept with the velocity axis, while the latter determines its curvature. A large value of a/Po is associated with a less prominent curvature of P-V curve, and a low efficiency of converting chemical energy derived from ATP hydrolysis into mechanical work [25]. On this basis, the nearly straight $\mathrm{P}-\mathrm{V}$ curve of cytoplasmic myosin versus actin cable sliding (Figures 12 and 13) suggests a very low efficiency of the chemo-mechanical energy conversion in cytoplasmic myosin molecules. The value of Vmax for cytoplasmic myosin versus actin cable sliding, on the other hand, is many times larger than that in skeletal and cardiac myosin versus actin cable sliding, suggesting that the maximum cycling rate of actinmyosin interaction is many times larger in the former than in the latter.

To explain the increasing degree of deviation of the P-V curves of skeletal and cardiac myosin versus actin cable sliding from the hyperbola with decreasing value of Po, we used the concept of $p$ and Poff. In contrast, the P-V curve of cytoplasmic myosin versus actin cable sliding was straight irrespective of the value of Po (Figures 11 and 12) [23]. This implies that, in cytoplasmic streaming, the probability 
of Poff is extremely small despite very small Po values. In other words, the value of $p$, representing the fraction of time in which a myosin head firmly attaches to actin to produce actin-myosin sliding, i.e. duty ratio [26], may be very large and close to unity. We think that the large duty ratio is essential for producing continuous cytoplasmic streaming with the ultra-high velocity.

\section{Possible Mechanism of the Ultra-high Cytoplasmic Streaming}

Based on the concept of duty ratio, there are three basic kinetic factors, i.e. (1) duty ratio of myosin head ( $p$ ), (2) ATPase rate per myosin head $(A)$, and (3) unitary sliding distance produced by a myosin head $(d)$. The value of $p$ is written as:

$$
p=(d \times A) / \mathrm{Vmax} \text {. }
$$

In the ATP-dependent actin-myosin sliding in skeletal muscle, $\mathrm{p}$ is $\sim 0.05$ [27,28], $A$ is $\sim 20 / \mathrm{s} /$ head [29], and Vmax is $\sim 3 \mu \mathrm{m} / \mathrm{s}$ [13]. Putting these values into the above equation, $d$ is obtained to be $\sim 8 \mathrm{~nm}$, a value consistent with the contraction model, in which myosin head power stroke is caused by change in myosin head configuration [30].

In the ATP-dependent actin-cytoplasmic myosin sliding, $p$ is assumed to be 0.25 , while $\mathrm{Vmax}$ is $50 \mu \mathrm{m} / \mathrm{s}$ [23]. The value of $d$ is unknown, but it is not $>20 \mathrm{~nm}$, if the size of myosin head is taken into consideration. From these values, above equation indicates that $A$ should be $\sim 425 / \mathrm{s} /$ head. The extremely high cyclic rate of actincytoplasmic myosin interaction, however, contradicts the result that actin-activated ATPase activity of cytoplasmic myosin in solution is $20 / \mathrm{s} /$ head, being similar to that in skeletal muscle myosin in solution [31].

The puzzling situation can be solved at least qualitatively by assuming a cooperative mechanical interaction between adjacent myosin heads extending from cytoplasmic myosin molecule attached to the same bead or the same cytoplasmic organelle. Since the value of $p$, i.e. duty ratio in cytoplasmic myosin head is close to unity, each cytoplasmic myosin head may have many chances of being pushed by adjacent myosin heads to result in rapid detachment from actin to restart its power stroke producing cytoplasmic streaming. This may markedly increase the cycling rate of actin-myosin interaction coupled with ATP hydrolysis in cytoplasmic myosin heads extending from the same bead or the same organelle.

This idea comes from the report that, if single skeletal muscle fibers are previously stretched to sarcomere length of $3 \mu \mathrm{m}$ with resting tension amounting $\leq 7 \%$ of Po, the maximum shortening velocity of tetanized fibers, as measured by the slack test, increases $\sim$ two fold [32]. As the resting tension decreases quickly at the beginning of applied release, the myosin heads attached to actin in tetanized fibers can be pushed in the direction of fiber shortening by the resting tension for an extremely short period of time. This possibility has already been discussed by us in a short review of cytoplasmic streaming [33].

\section{Conclusion}

The large duty ratio of cytoplasmic myosin head might produce mechanical synchronization and summation of power strokes between adjacent myosin heads to result in marked increase in the rate of cyclic interaction to greatly increase the velocity of actin-myosin sliding. Considering the extremely small Reynolds number for myosin head power stroke in the cytoplasm, together with a duty ratio close to unity, adjacent myosin heads on the same cytoplasmic organelle can effectively interact each other to produce the ultra-fast cytoplasmic streaming. Myosins are widely distributed in both amimal and plant kingdoms, to comprise a superfamily, in which skeletal and cardiac myosins and cytoplasmic myosin are classified into myosin II and myosin XI, respectively [34]. To reach full understanding of myosin molecules, it is essential to study similarities and differences among different myosin types.

\section{Acknowledgement}

We dedicate this article to the late Professor Eiji Kamitsubo for his contribution to our studies on the sliding between cytoplasmic myosin and actin cables using the centrifuge microscope.

\section{References}

1. Kamiya N, Kuroda K (1956) Velocity distribution of the protoplasmic streaming in Nitella cells. Bot Mag 69: 544-554.

2. Kamitsubo E (1966) Motile protoplasmic fibrils in cells of Characeae. Proc Japan Acad 42: 640-643.

3. Nagai R, Rebhum I (1979) J Ultrastruct Res 14: 571-589

4. Kersey YM, Wessels NK (1976) Localization of actin filaments in internodal cells of characean algae. A scanning and transmission electron microscope study. J Cell Biol 68: 264-275.

5. Kato T, Tonomura Y (1977) Identification of myosin in Nitella flexilis. J Biochem 82: 777-782.

6. Nagai R, Hayama T (1979) Ultrastructure of the endoplasmic factor responsible for cytoplasmic streaming in Chara internodal cells. J Cell Sci 36: 121-136.

7. Tazawa M, Shimmen T (1987) Cell motility and ionic relations in characean cells as revealed by internal perfusion and cell models. Int Rev Cytol 109: 259312.

8. Williamson RE (1975) Cytoplasmic streaming in Chara: a cell model activated by ATP and inhibited by cytochalasin B. J Cell Sci 17: 655-668.

9. Williamson RE, Ashley CC (1982) Free Ca2+ and cytoplasmic streaming in the alga Chara. Nature 296: 647-651.

10. Tominaga $Y$, Shimmen T, Tazawa M (1983) Control of cytoplasmic streaming by extracellular $\mathrm{Ca} 2+$ in permeabilized Nitella cells. Protoplasma 116: 75-77.

11. Shimmen T, Yano M (1984) Active sliding movement of latex beads coated with skeletal muscle myosin on Chara actin bundles. Protoplasma 121: 132-137.

12. Sheetz MP, Spudich JA (1983) Nature 303: 31-35.

13. Oiwa K, Chaen S, Kamitsubo E, Shimmen T, Sugi H (1990) Steady-state forcevelocity relation in the ATP-dependent sliding movement of myosin-coated beads on actin cables in vitro studied with a centrifuge microscope. Proc Natl Acad Sci USA 87: 7893-7897.

14. Higashi-Fujime S, Ishikawa R, Iwasawa H, Kagami O, Kurimoto E, et al. (1995) The fastest-actin-based motor protein from the green algae, Chara, and its distinct mode of interaction with actin. FEBS Lett 375: 151-154.

15. Yamamoto Y, Hamada S, Kashiyama T (1999) Cell Mol Life Sci 56: 226-232.

16. Tsuchiya T, Tanaka H, Shirakawa I, Karr T, Sugi H (1998) Evidence for the essential role of myosin subfragment-2 in the ATP-dependent actin-myosin sliding in muscle contraction. Jpn J Physiol 48: 383-387.

17. Edman KAP (1988) Contractile performance of skeletal muscle fibres. J Physio 484: 301-321

18. Iwamoto $H$, Sugaya $R$, Sugi $H$ (1990) Force-velocity relation of frog skeleta muscle fibres shortening under continuously changing load. J Physiol 422 185-202.

19. Sugi H, Kobayashi T, Gross T, Noguchi K, Karr T, et al. (1992) Contraction characteristics and ATPase activity of skeletal muscle fibers in the presence of antibody to myosin subfragment 2. Proc. Natl. Acad. Sci. USA 89: 6134-6137.

20. Ohno T, Arao T, Chaen S, Sugi H (2017) J Nanomed Nanotechno 8: 1000428.

21. Sugiura S, Yamashita H, Sata M. Momomura S, Serizawa T, et al. (1995) Force-velocity relations of rat cardiac myosin isozymes sliding on algal cell actin cables in vitro. Biochim. Biophys Acta 1231: 69-75. 
Citation: Chaen S, Sugi H (2018) Mechanism of Ultra-Fast Actin-Myosin Sliding Producing Cytoplasmic Streaming in Giant Algal Cell, Studied Using the Centrifuge Microscope. J Material Sci Eng 7: 426. doi: 10.4172/2169-0022.1000426

22. Huxley AF (1957) Muscle structure and theories of contraction. Prog Biophys Biophys Chem 7: 255-318.

23. Chaen S, Inoue J, Sugi H (1995) The force-velocity relationship of the ATPdependent actin-myosin sliding causing cytoplasmic streaming in algal cells studied using a centrifuge microscope. J Exp Biol 198: 1021-1027.

24. Woledge RC, Curtin NA, Homsher E (1985) Energetic Aspects of Muscle Contraction, Academic Press, London

25. Woledge RC (1968) The energetics of tortoise muscle. J Physiol 197: 685-707.

26. Howard J (1997) Molecular motors: structural adaptations to cellular functions. Nature 389: 561-567.

27. Ueyda TPQ, Kron SJ, Spudich JA (1990) Myosin step size: estimation from slow sliding movement of actin over low densities of heavy meromyosin. J Mol Biol 214: 699-710.

28. Finer JT, Simmons RM, Spudich JA (1994) Single myosin molecule mechanics: piconewton forces and nanometre steps. Nature 368: 113-119.
29. Toyoshima Y, Kron SJ, Spudich JA (1990) The myosin step size: measurement of the unit displacement per ATP hydrolyzed in an in vitro assay. Proc Natl Acad Sci USA 87: 7130-7134.

30. Huxley AF, Simmons RM (1971) Proposed mechanism of force generation in striated muscle. Nature 233: 533-538.

31. Tanimura A, Higashi-Fujime S (2002) Force-velocity relationships in actinmyosin interactions causing cytoplasmic streaming in algal cells. J Muscle Res Cell Motil 23: 185.

32. Edman KAP (1979) The velocity of unloaded shortening and its relation to sarcomere length and isometric force in vertebrate muscle fibres. J Physiol 291: 143-159.

33. Sugi H, Chaen S (2003) Force-velocity relationships in actin-myosin interactions causing cytoplasmic streaming in algal cells. J Exp Biol 206: 19711976.

34. Sellers J (S2000) Myosins: a diverse superfamily. Biochim Biophys Acta 1496: 3-22. 